\title{
Opportunities to expand access to mental health services: A case for the role of online peer support communities
}

\author{
Rutvij Merchant ${ }^{1}$ (D) - Aleah Goldin ${ }^{2}$. Deepa Manjanatha ${ }^{3} \cdot$ Claire Harter $^{2}$. \\ Judy Chandler ${ }^{2}$ Amanda Lipp ${ }^{4} \cdot$ Theresa Nguyen $^{5} \cdot$ John A. Naslund ${ }^{6}$
}

Accepted: 26 January 2022 / Published online: 28 February 2022

(c) The Author(s), under exclusive licence to Springer Science+Business Media, LLC, part of Springer Nature 2022

\begin{abstract}
This study investigated whether with disruptions in care due to the COVID-19 pandemic, persons who self-identified as living with a mental health condition increased their usage of an online peer support community. We also explored whether study participants who self-reported usage of online peer support communities were interested in using these communities to connect with evidence-based interventions and mental health services. This study employed a cross-sectional online survey design. The survey was disseminated by the Inspire online peer support community and Mental Health America through various social media channels. Descriptive and inferential analyses were conducted to summarize participant demographics, investigate patterns of use of online peer support communities, and interest in accessing additional programs through these platforms. There were 369 survey respondents, with a mean age of $49(\mathrm{SD}=15.6)$, of which $77 \%$ were female. Most respondents self-reported having depression (34\%), post-traumatic stress disorder (25\%), and anxiety-related conditions (20\%). The number of respondents who reported accessing online peer support platforms multiple times a day appeared to double after March 2020. We also found an overall positive association between frequency of community use and self-reported benefit to mental health. Approximately $81 \%$ of respondents expressed interest in accessing mental health services via an online peer support community. Persons who self-report living with mental health conditions and who engage in online peer support communities expressed interest in accessing evidence-based interventions via these online platforms. Participants were most interested in services related to enhancing coping mechanisms and skills, enabling overall wellbeing, and accessing therapy.
\end{abstract}

Keywords Digital peer support $\cdot$ Mental health $\cdot$ Early intervention $\cdot$ Online communities

Rutvij Merchant

rutvij.merchant@icahn.mssm.edu

1 Icahn School of Medicine at Mount Sinai, New York, NY 10029, USA

2 Inspire, Arlington, VA 22201, USA

3 Harvard TH Chan School of Public Health, Harvard University, Boston, MA 02115, USA

4 Center for Applied Research Solutions Inc, Santa Rosa, CA 95404-410, USA

5 Mental Health America, Alexandria, VA 22314, USA

6 Department of Global Health and Social Medicine, Harvard Medical School, Boston, MA 02115, USA 


\section{Introduction}

Online peer support communities offer persons living with mental health conditions the opportunity to exchange knowledge and social support with peers who share similar lived experiences [1]. These communities (also called online health communities, peer-to-peer support platforms, or peer support networks) provide a virtual space for patients and caregivers to contribute and share information, and in the process, construct vast searchable and interactive archives [2]. These living digital libraries contain information not typically available within the formal healthcare landscape and are especially important since many persons facing mental health challenges frequently search online prior to and after seeking formal care $[3,4]$. A recent survey of persons with co-occurring physical and mental health conditions found that $63 \%$ of adults ages 35-64 reported not knowing how to bring up mental health concerns to a healthcare provider and $64 \%$ were uncertain if their mental health concerns were significant enough to mention to a healthcare provider [5]. In such cases, persons with mental health conditions may turn to online peer support communities to read and ask for advice from peers, overcoming hurdles through mutual sharing, and learning from the experiential knowledge of others [6].

Online peer support communities have existed for over two decades, and have mostly been focused on chronic conditions and various forms of cancer [7, 8], but there is now mounting interest in the role of such platforms for mental health services [9-11]. Some scholars have begun encouraging healthcare providers to visit these communities and even recommend that healthcare providers refer patients to them, since this may facilitate shared decision-making $[12,13]$. Other studies have also suggested that online peer support communities could be beneficial in helping individuals find their desired forms of care, negotiating healthcare provider relationships, and navigating health systems $[6,14,15]$. It also has been suggested that reading, commenting on, and publishing posts on these platforms may allow persons living with mental health conditions to feel more empowered, socially connected, and informed about their decision-making [1, 16].

The COVID-19 pandemic brought significant disruptions to conventional mental health care, resulting in a rapid transition to remote consultations (telephone and video) with widespread restrictions on in-person visits [17]. With the increasing reliance on digital technology for access to mental health services, there has been renewed interest in understanding the role that online peer support communities could play in bridging service gaps. For instance, online peer support communities can offer individuals alternative avenues to seek support when confronted with barriers to accessing formal care, such as costs, transportation, and stigma. This is especially important as the pandemic has exacerbated risks for mental health concerns [18]. With increasing demand for mental health services coupled with growing adoption of digital technology, online peer support communities may be well-positioned to collaborate with conventional care to expand access and reach of evidence-based services. Therefore, in this exploratory study, we employed online survey methods to better understand the role of online peer support communities from the perspectives of persons who self-report living with mental health conditions.

Specifically, we investigated whether with the onset of the pandemic, persons who selfidentified as living with a mental health condition reported increased usage of an online peer support community. We hypothesized that a decrease in access to in-person services and rapid transition to remote platforms might have resulted in an increase in usage of online peer support communities. We also explored whether persons who self-identified as 
living with a mental health condition and who self-reported usage of online peer support communities would be interested in connecting with additional evidence-based resources and interventions through these online communities.

\section{Methods}

\section{Study Design}

This study employed a cross-sectional online survey design, distributed by Inspire, an online support community for patients and caregivers. Inspire has 2.5 million members and partners with Mental Health America, a leading community-based nonprofit dedicated to addressing the needs of those living with mental health conditions, to co-manage online peer support communities focused on mental health. As part of this study, Inspire and Mental Health America disseminated an anonymous online survey through various online channels, social media platforms, and listservs as described below. No identifiable or personal details were collected from survey respondents; therefore, this study did not meet the federal definition of human subjects research as determined by the NIH Human Subjects Research Decision Tool from the Harvard Medical School Institutional Review Board.

\section{Participants}

Eligible respondents self-reported living with a mental health condition, being Englishspeaking, $\geq 18$ years of age, and indicated interest and willingness to complete the survey.

\section{Survey and Study Procedures}

Participation in the survey was voluntary, and survey questions were administered through Alchemer, an online survey tool. Responses were collected anonymously to protect respondents' identities. In September 2020, an invitation to participate in the survey was shared via email listservs, newsletters, and social media channels. Distribution of the survey included emails to members of the following communities on Inspire: Mental Health America, iFred Anxiety and Depression, and Schizophrenia Spectrum Support. The survey was also disseminated on Mental Health America's Twitter and LinkedIn as well as Instagram posts from Motherhood Understood, a postpartum mental health platform with connections to both Inspire and Mental Health America. The survey comprised 35 questions and took approximately 15-20 min to complete. All questions were optional, and upon completion, respondents could choose to input their email address to be entered into a raffle to win one of three $\$ 25$ Amazon gift cards. Entering the raffle was not connected to collection of the survey responses to ensure anonymity. Subsequently, the de-identified survey data was exported to a csv file for exploratory analysis.

\section{Data Collection}

As part of the survey, respondents were asked about their geographic location, age, gender, race and ethnicity, self-reported mental health diagnosis, education level, employment status, 
sexual orientation, and living status. Respondents were also asked a series of questions to shed light on the following three key points: First, respondents were asked about their general use of online peer support platforms, including their frequency of use of these platforms as well as their use of these platforms before and after the onset of the COVID-19 pandemic. Next, respondents were asked about positive or negative impacts on their mental health from using online peer support communities and potential concerns or perceived risks with using these platforms. Lastly, respondents were asked if they would be interested in accessing additional evidence-based services or mental health interventions through online peer support communities, and if so, they could select from a pre-populated list with a range of options given (e.g., programs to help navigate the healthcare system, programs to help with managing symptoms, programs to help reduce usage of drugs, alcohol, or tobacco, etc.).

\section{Data Analysis}

Descriptive statistics were used to summarize respondent demographic characteristics and responses to the survey questions. Regression analysis was used to examine the association between the frequency of platform use and self-reported benefit to mental health. A linear probability model provided general insights about the relationship between these two variables, after controlling for age, education, gender, and race of the respondents. STATA/IC software version 16.1 for Windows was used for all statistical analyses.

\section{Results}

\section{Characteristics of Survey Respondents}

In total, 369 persons living with a mental health condition responded to the online survey, with a mean age of $49(\mathrm{SD}=15.6)$. Approximately $77 \%$ were female, and $85 \%$ identified as being non-Hispanic white. Respondents had a range of self-reported mental health diagnoses, with the most prominent being: depressive conditions (34\%), post-traumatic stress disorder [PTSD] (25\%), and anxiety-related conditions (20\%). Fifty percent of respondents reported having a college degree or higher, $41 \%$ reported some college (including $15 \%$ with an associate's or technical degree), and the remaining $9 \%$ reported having high-school education or less. A detailed summary of sample characteristics is included in Table 1.

\section{Use of Online Peer Support Communities Before and After the Onset of the COVID-19 Pandemic}

As illustrated in Fig. 1, the number of respondents who reported accessing online peer support communities multiple times a day appeared to double after March 2020, from 13 to $27 \%$. There was also a $50 \%$ increase in the number of respondents who accessed online peer support communities at least once a day (from 42 to $65 \%$ ) and a $15 \%$ increase in the number of respondents who accessed online peer support platforms at least once a week (from 80 to 92\%) from prior to the onset of the pandemic. 
Table 1 Respondents' demographic characteristics

Characteristic

Demographic Characteristics

Gender

Female

$$
283
$$

Male

Other

Race/Ethnicity

Non-White

White

Age

$18-30$

$31-60$

61-84

Employment

Full-time employment

Part-time employment

Student

Not employed

On disability or medical leave

Volunteer, retired, or caregiver

Preferred not to respond

Other

Living situation

Live alone

Live with family

Live with partner (e.g., spouse, significant other)

Group home or supported housing

Unsheltered/homeless

Other (e.g., retirement community, student housing, 10 etc.)

Education

Less than high school

Graduated high school

Associate's degree or technical degree

Some college, no degree

4-year college degree

Post-graduate degree (Master's, Ph.D., M.D.)

Clinical Characteristics

Self-reported mental health condition diagnoses breakdown

ADHD/ADD

Anxiety Condition

Bipolar Condition

Borderline Personality Condition

Delusional Condition
76.69

.08

15.45

13

8.5

15

11.4

1.1

7.6

17.8

9 
Table 1 (continued)

\begin{tabular}{lll}
\hline Characteristic & $\begin{array}{l}\text { Total number of respondents, } \\
\mathbf{N = 3 6 9}\end{array}$ & $\begin{array}{l}\text { Percent of total } \\
\text { respondents }\end{array}$ \\
\hline Depressive Conditions & 189 & 51.22 \\
Psychotic Condition & 9 & 2.44 \\
PTSD & 105 & 28.46 \\
Schizoaffective Condition & 18 & 4.88 \\
Schizophrenia Spectrum Conditions & 16 & 4.34 \\
Schizotypal Personality Condition & 4 & 1.08 \\
Schizophreniform Condition & 1 & .27 \\
Other (i.e., disordered eating, learning disability, & 13 & 3.52 \\
$\quad$ OCD, PMDD, PPD, etc.) & & \\
\hline
\end{tabular}

\section{Association Between Self-reported Benefit to Mental Health and Frequency of Use of Online Peer Support Communities}

As outlined in Fig. 2, respondents who used online peer support platforms at least once a day or at least once a week, were more likely to self-report that these platforms benefited their mental health.

In findings from a linear probability model, there was a positive association between frequency of use of online peer support communities (after the onset of the pandemic) and

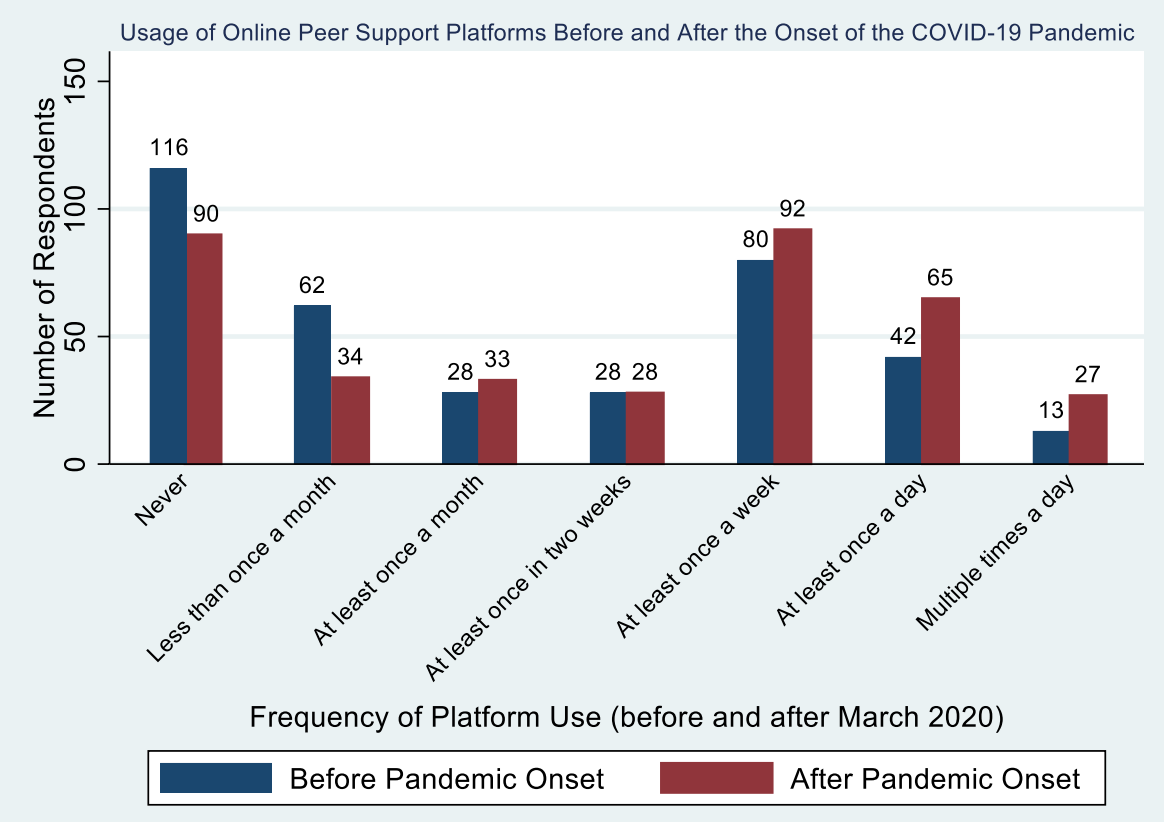

Fig. 1 Respondents' self-reported use of online peer support platforms before and after the onset of the COVID-19 pandemic 


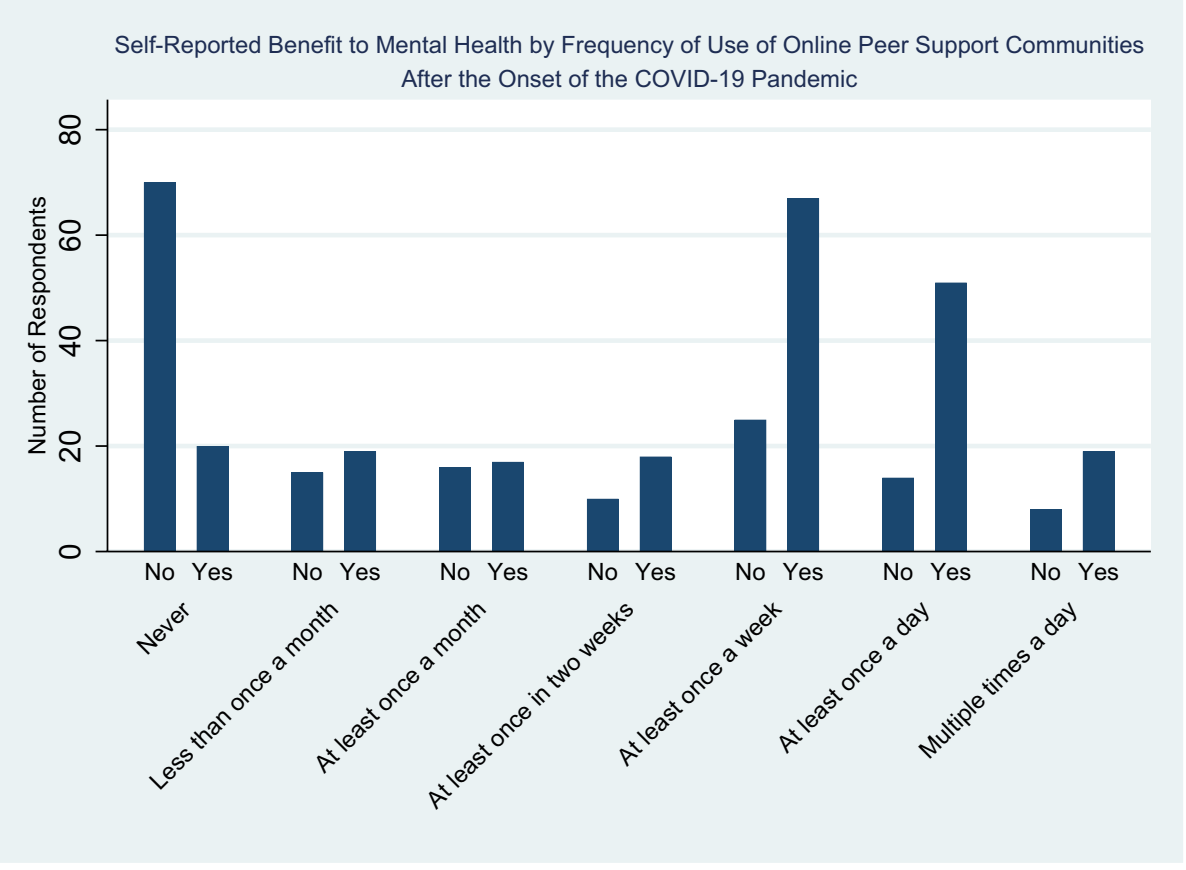

Fig. 2 Respondents' self-reported benefit to mental health and frequency of use of online peer support communities

self-reported benefit to mental health after adjusting for the effect of age, education, gender, and race. The results showed that a one unit increase in our categorization of frequency of platform use (reference the Appendix for specific categories) was associated with an $11.5 \%$ increase in the probability $(t(363)=11.43, p<.001)$ of a respondent reporting that the platform benefited their mental health.

\section{Interest in Accessing Psychoeducational and Mental Health Services Through Online Peer Support Communities}

Overall, 298 out of the 369 respondents (approximately $81 \%$ of the respondents) expressed interest in accessing psychoeducational and mental health services via online peer support communities. Respondents were asked a follow-up question about the specific types of services they may be interested in accessing, and Fig. 3 provides a breakdown of these responses.

Respondents expressed the most interest in online peer support communities collaborating with formal care to connect to services oriented around wellbeing (178 respondents; $48 \%$ ), services focused on coping mechanisms and skills (177 respondents, 48\%), and online therapy sessions tailored for their specific mental health conditions (174 respondents, $47 \%$ ). Additionally, a smaller portion of respondents expressed interest in online peer support communities connecting them with support services to aid them in navigating the healthcare system (63 respondents, 17\%) or managing substance use (specifically, drugs, alcohol, and cigarettes) (50 respondents, 14\%). 


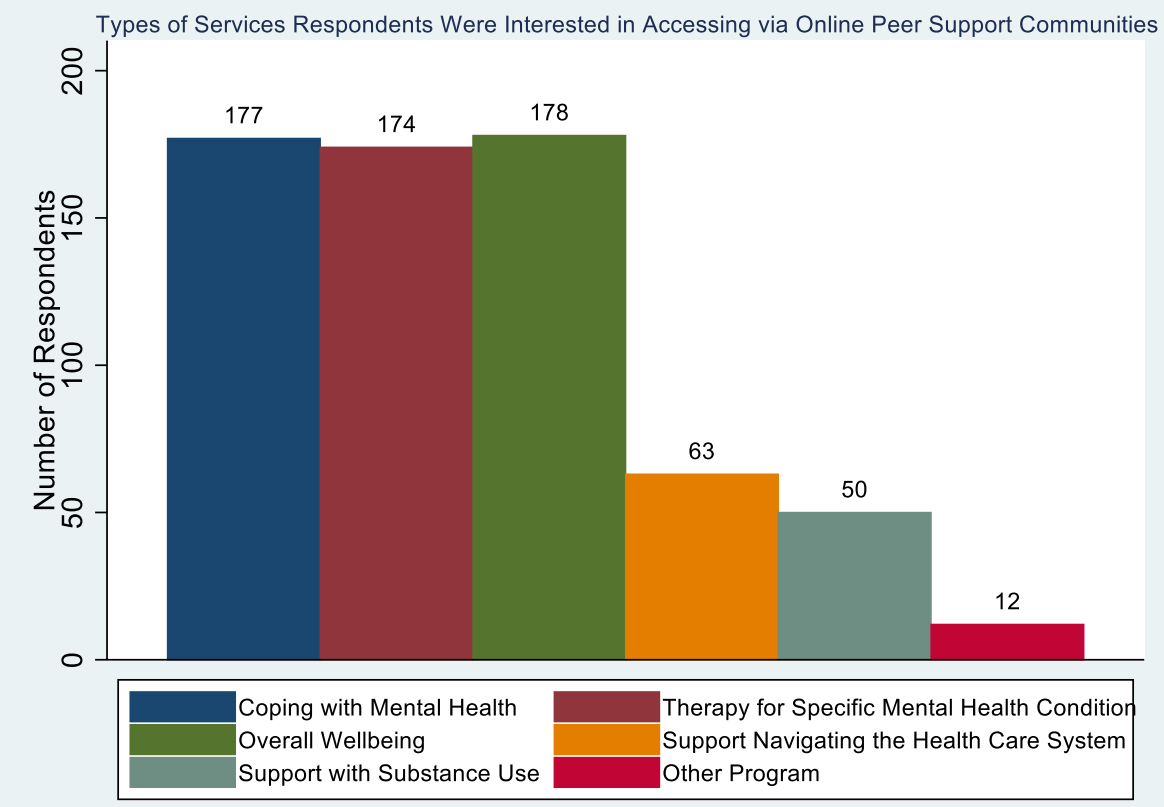

Fig. 3 The types of psychoeducational and mental health services that respondents expressed interest in accessing via online peer support communities

Through open responses, 12 respondents offered ideas for other types of services and more specific offerings that were not listed on the survey. These ideas ranged from specifying one-on-one CBT-based virtual therapy to professional-led group therapy. In this openended manner, a few respondents also expressed interest in online support services for disordered eating $(n=1)$ and $(n=1)$ requested services that could aid with proper eating, physical health, exercise, and healthy weight loss support. Several of these respondents $(n=4)$ expressed interest in online mental health services that could aid with chronic pain, with one $(n=1)$ expressing interest specifically in condition-tailored support (i.e., Premenstrual dysphoric disorder (PMDD)). Another individual expressed interest in online services that could aid with general life management tasks, for example, guiding users through the process of choosing from insurance plans.

\section{Discussion}

This study builds upon previous research examining the role of online peer support communities for persons living with mental health conditions $[19,20]$. The dramatic shift to virtual care necessitated by the COVID-19 pandemic has substantially altered the delivery of mental health services [21] and has prompted us to inquire how we can better meet the needs of persons living with mental health conditions. As part of this inquiry, we examined the patterns of use evident within online communities during the pandemic and explored 
whether there may be interest among persons living with mental health conditions to access evidence-based resources or interventions through these digital spaces.

Importantly, our findings highlight that some persons living with mental health conditions appear interested in accessing additional evidence-based programs, such as psychoeducational resources and other interventions via online peer support communities. This could lead to innovative collaborations between online peer support communities and conventional care, as these persons tend to be most interested in evidence-based interventions and services related to enhancing coping mechanisms and skills, enabling overall wellbeing, and supporting access to therapy. Encouragingly, a recent systematic review of digital peer support interventions for persons with a lived experience of a serious mental illness found that peer-to-peer networks combined with evidence-based practice interventions reported statistically significant improvements in psychiatric symptoms [10]. Taken together, these findings suggest that online peer support communities should be explored further as a potential avenue for collaboration, particularly for delivery of effective, evidence-based programs, and as a tool that might help bridge gaps in service delivery.

This is especially important to research further as the expansion of teletherapy alone, as necessitated by the COVID-19 pandemic, has not brought an influx of new patients into the care delivery system. According to data from recent TIME/Harris Poll national surveys, only $5 \%$ of 3,214 respondents indicated they received mental health services for the first time during the height of the COVID-19 crisis [21]. This suggests that despite the growth in digital methods of care delivery, issues in accessing support services and evidence-based interventions persist. A recent RAND Corporation report found that only $45 \%$ of people with a mental illness in the US received any mental health treatment in 2019 [22]. The gap in access is more pronounced among particular segments of the population. Despite similar levels of mental health care need, racial/ethnic minorities in the United States are half as likely to use mental health care as non-Hispanic Whites [22]. As such, researchers and healthcare providers need to continue exploring a range of mechanisms for facilitating access. In this context, the potential of online peer support communities as avenues for collaboration, complementary support, and delivery of evidence-based services should be further investigated.

Peer support is seen as an essential recovery service for people with mental health conditions globally [23] and is currently being implemented in some primary care settings as a patient-centered approach [24]. In the United States, the recently passed PEERS Act provides Medicare coverage of peer support services for individuals with mental illness and/or substance use disorders who are receiving integrated behavioral health services [25]. This legislation ensures peer support specialists are recognized and compensated, incentivizing the development of novel methods that deliver formal peer services online. Such peer support, whether in-person/digital or formal/informal, may encourage persons to initially seek out care and continue with care [26]. The growing recognition of the potential benefits of peer support - and the digital communities built to encourage such interactions - could help scholars and practitioners develop mental healthcare delivery models that align conventional care and online support on a larger scale. Moreover, collaborations between existing digital peer support communities and formal mental health services could potentially mitigate some barriers to access and streamline interventions, as many patients already complement traditional health care trajectories by exchanging information about conditions and guidance in treatments via online peer support communities [7].

Further research is required to determine best practices in developing collaborative care delivery models that align digital peer support communities with formal mental health services. This study suggests that persons living with mental health conditions may be 
interested in the evolution of such models and any further research should closely involve target audiences across the development, testing, and implementation stages.

\section{Limitations}

More research is needed to determine the often invisible and unrecognized ways in which online peer support communities are already part of the care system and could further complement formal care. Future studies are needed that investigate this topic longitudinally and across a wide range of online peer support communities, especially given the differences in reach and target audiences across the diversity of online platforms. Investigating this topic across other online peer support communities is vital as our study contained a sample who tended to be female, non-Hispanic white, older, and highly educated. As such, it is critical to expand the reach of this exploratory research to investigate how interest in evidencebased interventions through these communities differs by gender, race, age, education, and socioeconomic demographics.

More research is also needed to explore the specific types and mediums of evidencebased interventions and resources that may be of interest and how they should be distributed in these communities according to persons living with mental health conditions. This study did not aim to explore these specifics but rather set the stage for future investigations into the development and dissemination of such resources, particularly for those at the earliest stages of intervention. An important area of future research also involves examining how evidence-based interventions offered through online peer support communities can offer safe virtual environments for diverse population groups and democratize access to care for underserved racial and ethnic minority groups.

Our study found that respondents who use online peer support communities at least once a day or at least once a week are more likely to self-report that these communities contributed to benefits to their mental health. This finding is vulnerable to selection bias as respondents who see benefits in the use of online peer support platforms are more likely to use the platform more frequently. This can be addressed in future research by engaging a larger sample of online peer support community members who exhibit diverse patterns of community use. If the finding is replicated among this larger and more diverse group, it would offer further evidence supporting an association between frequency of online peer support community use and self-reported benefits to mental health.

\section{Conclusion}

We found that the onset of the pandemic was associated with an increased usage of online peer support communities and an overall positive association between frequency of platform use and self-reported benefit to mental health. Our findings also highlight that persons living with mental health conditions are interested in accessing evidence-based interventions and resources via online peer support communities. Persons were most interested in services related to enhancing coping mechanisms and skills, enabling overall wellbeing, and therapy.

Our exploratory study suggests that online peer support communities can potentially play a key role in helping to better meet the needs of persons living with mental health conditions. The study also sets the stage for further research to determine best practices in developing care delivery models in which digital peer support communities and formal 
care collaborate more closely to support access to evidence-based mental health resources and services.

Data Availability Statement The data that support the findings of this study are available from the corresponding author upon reasonable request.

\section{Declarations}

Ethics Approval All procedures performed in the study involving human participants were in accordance with the ethical standards of the Helsinki Declaration and its later amendments or comparable ethical standards. No identifiable or personal details were collected from survey respondents; therefore, this study did not meet the federal definition of human subjects research as determined by the NIH Human Subjects Research Decision Tool from the Harvard Medical School Institutional Review Board.

Conflict of Interest Statement The authors declare that they have no conflict of interest.

\section{References}

1. Naslund JA, Aschbrenner KA, Marsch LA, Bartels SJ. The future of mental health care: peer-to-peer support and social media. Epidemiol Psychiatr Sci. 2016;25(2):113-22. https://doi.org/10.1017/S2045796015001067.

2. Gupta T, Schapira L. Online communities as sources of peer support for people living with cancer: a commentary. J Oncol Pract. 2018;14(12):725-30. https://doi.org/10.1200/JOP.18.00261.

3. Pretorius C, Chambers D, Coyle D. Young people's online help-seeking and mental health difficulties: Systematic narrative review. J Med Internet Res. 2019;21(11): e13873. https://doi.org/10.2196/13873.

4. Hanley T, Prescott J, Gomez KU. A systematic review exploring how young people use online forums for support around mental health issues. J Ment Health. 2019;28(5):566-76. https://doi.org/10.1080/ 09638237.2019.1630725.

5. Mental Health America. Creating better care for adults with comorbid chronic conditions. Mental Health America. 2020. https://www.mhanational.org/sites/default/files/Creating\%20Better\%20Care\%20for\%20 Adults\%20with\%20Comorbid\%20Chronic\%20Conditions\%20-\%20Report\%20ONLY\%201.8.21.pdf. Accessed 15 Jul 2021.

6. Foster D. 'Keep complaining till someone listens': Exchanges of tacit healthcare knowledge in online illness communities. Soc Sci Med. 2016;166:25-32. https://doi.org/10.1016/j.socscimed.2016.08.007.

7. Johansson V, Islind AS, Lindroth T, Angenete E, Gellerstedt M. Online communities as a driver for patient empowerment: Systematic review. J Med Internet Res. 2021;23(2): e19910. https://doi.org/10. 2196/19910.

8. Ziebland SUE, Wyke S. Health and illness in a connected world: how might sharing experiences on the internet affect people's health? Milbank Q. 2012;90(2):219-49. https://doi.org/10.1111/j.14680009.2012.00662.x.

9. Ali K, Farrer L, Gulliver A, Griffiths KM. Online peer-to-peer support for young people with mental health problems: a systematic review. JMIR Ment Health. 2015;2(2):4418. https://doi.org/10.2196/ mental.4418.

10. Fortuna KL, Naslund JA, LaCroix JM, Bianco CL, Brooks JM, Zisman-Ilani Y, Deegan P. Digital peer support mental health interventions for people with a lived experience of a serious mental illness: systematic review. JMIR Ment Health. 2020;7(4): e16460. https://doi.org/10.2196/16460.

11. Ridout B, Campbell A. The use of social networking sites in mental health interventions for young people: systematic review. J Med Internet Res. 2018;20(12):12244. https://doi.org/10.2196/12244.

12. Petrič G, Atanasova S, Kamin T. Impact of social processes in online health communities on patient empowerment in relationship with the physician: emergence of functional and dysfunctional empowerment. J Med Internet Res. 2017;19(3): e74. https://doi.org/10.2196/jmir.7002.

13. Solberg LB. The benefits of online health communities. AMA J Ethics. 2014;16(4):270-4. https:// doi.org/10.1001/virtualmentor.2014.16.4.stas1-1404. 
14. Wicks P, Massagli M, Frost J, Brownstein C, Okun S, Vaughan T, Heywood J. Sharing health data for better outcomes on PatientsLikeMe. J Med Internet Res. 2010;12(2): e19. https://doi.org/10. 2196/jmir. 1549.

15. Willis E. Patients' self-efficacy within online health communities: facilitating chronic disease selfmanagement behaviors through peer education. Health Commun. 2016;31(3):299-307. https://doi. org/10.1080/10410236.2014.950019.

16. Newman MW, Lauterbach D, Munson SA, Resnick P, Morris ME. It's not that I don't have problems, I'm just not putting them on Facebook: challenges and opportunities in using online social networks for health. Proc Assoc Comput Mach Conf Comput Support Cooper Work 2011;34150. https://doi.org/10.1145/1958824.1958876.

17. Bojdani E, Rajagopalan A, Chen A, Gearin P, Olcott W, Shankar V, DeLisi LE. COVID-19 pandemic: impact on psychiatric care in the United States. Psychiatry Res. 2020;289. https://doi.org/ 10.1016/j.psychres.2020.113069.

18. Panchal N, Kamal R, Cox C, Garfield R. The implications of COVID-19 for mental health and substance use. Kaiser Family Foundation. 2021. https://www.kff.org/coronavirus-covid-19/issue-brief/ the-implications-of-covid-19-for-mental-health-and-substance-use/. Accessed 17 Feb 2022.

19. Low DM, Rumker L, Talkar T, Torous J, Cecchi G, Ghosh SS. Natural language processing reveals vulnerable mental health support groups and heightened health anxiety on Reddit during COVID19: Observational study. J Med Internet Res. 2020;22(10):e22635. https://doi.org/10.2196/22635.

20. Hwang J, Toma CL, Chen J, Shah DV, Gustafson D, Mares ML. Effects of web-based social connectedness on older adults' depressive symptoms: a two-wave cross-lagged panel study. J Med Internet Res. 2021;23(1):e21275. https://doi.org/10.2196/21275

21. Ducharme J. Teletherapy aimed to make mental health care more inclusive. The data show a different story. TIME. 2021. https://time.com/6071580/teletheraphy-mental-health/.

22. McBain, Ryan K., Nicole K. Eberhart, Joshua Breslau, Lori Frank, M. Audrey Burnam, Vishnupriya Kareddy, Molly M. Simmons, How to Transform the U.S. Mental Health System: Evidence-Based Recommendations. Santa Monica, CA: RAND Corporation. RR-A889-1. 2021. As of 28 Aug 2021: https:// www.rand.org/pubs/research_reports/RRA889-1.html.

23. World Health Organization. One-to-one peer support by and for people with lived experience. QualityRights guidance module. Geneva: World Health Organization; Licence: CC BY-NC-SA 3.0 IGO. 2019. https://apps.who.int/iris/bitstream/handle/10665/329591/9789241516785-eng.pdf. Accessed 15 Feb 2022.

24. Shepardson, R. L., Johnson, E. M., Possemato, K., Arigo, D., and Funderburk, J. S. Perceived barriers and facilitators to implementation of peer support in Veterans Health Administration Primary Care-Mental Health Integration settings. Psychol Serv. 2019;16(3):433. https://doi.org/10.1037/ ser0000242.

25. National Association of State Mental Health Program Directors, Mental Health America, the Depression and Bipolar Support Alliance, the National Association of Peer Supporters and the Association for Behavioral Health and Wellness. Leading mental health organizations applaud the introduction of the PEERS ACT of 2020 to allow participation of peer support specialists in behavioral health integration services in Medicare. Mental Health America. 2020. https:// mhanational.org/sites/default/files/Statement\%20on\%20Peers\%20Act\%20of\%202020revised2.pdf. Accessed 28 Aug 2021.

26. Marill MC, Beyond twelve steps, peer-supported mental health care. Health Aff. 2019;38(6). https:// doi.org/10.1377/hlthaff.2019.00503.

Publisher's Note Springer Nature remains neutral with regard to jurisdictional claims in published maps and institutional affiliations.

Rutvij Merchant is a medical student at the Icahn School of Medicine at Mount Sinai. His research interests lie at the intersection of mental health delivery and health system reform. He is also interested in the use of digital technology to deliver mental health services for populations living in low-resource settings. He has earned a BA in Political Science from Northwestern University and an MPH from the Harvard T.H Chan School of Public Health.

Aleah Goldin leads studies in the healthcare and insurance space to better understand unmet needs, motivations, and constraints. She earned a B.A. in Global Health from University of Richmond and a M.F.A. in Creative Writing and M.A. in Composition and Rhetoric from University of Alabama. She works as 
an adjunct professor at American University and has taught courses on illness narratives and multimodal storytelling.

Deepa Manjanatha is currently a research program manager motivated to address sources of social inequality and system inefficiency. She is especially interested in improving health access, integration of care and preventive services, and positive health and wellbeing at the intersection of gender, race, and mental health through community-based, systemic, and technology aided interventions targeting the social determinants of health. Deepa has earned a B.A. in Economics from UC Berkeley and an MPH from the Harvard T.H Chan School of Public Health.

Claire Harter is a research manager performing targeted social listening and digital analytics in the healthcare field. Her research background includes secondary and primary research in marketplace discourse, social and online identities, and doctor-patient communication. In addition to performing research, Claire is herself an active patient and advocate for narcolepsy, HHT, and chronic pain. Claire earned her MA in Linguistics from the University of Florida in 2015 with a focus in discourse analysis and sociolinguistics. Claire lives in Wichita, Kansas, with her husband and four pet rats.

Judy Chandler, MPH, CHES currently serves as the Partnerships Director at Inspire. She works with Inspire's $100+$ non-profit advocacy partners to build and grow online support communities that connect patients and caregivers with valuable sources of information and support. Before joining the Inspire team in 2014, Judy's career focused on developing educational programs and content for several non-profit organizations. She holds a Master's Degree in Public Health from George Washington University and is a Certified Health Education Specialist.

Amanda Lipp is a documentary filmmaker, consultant, and public speaker passionate about mental health and social entrepreneurship. She has produced over 70 short documentary films about mental health, and given over 150 talks sharing resources, films, and her own personal story. She serves on the Mental Health Advisory Panel at Google, and was appointed to the Interdepartmental Serious Mental Illness Committee (ISMICC) created by Congress to advise on federal mental health policy. Amanda formerly served on the board of the National Alliance on Mental Illness (NAMI). As a Research Associate for the Center for Applied Research Solutions, Amanda works on state and federal initiatives in behavioral health and LGBTQ funded by the Department of Health Care Services (DHCS) and the Substance Abuse and Mental Health Services Administration (SAMHSA). Amanda previously worked at the California Mental Health Services Authority (CalMHSA). Amanda graduated from UC Davis with a B.S. in Human Development.

Theresa Nguyen is Mental Health America's (MHA) Chief Program Officer and Vice President of Research and Innovation, Theresa works to improve access to mental health care through data and digital based innovations. Her areas of special interest include prevention, early intervention, education, and building a full recovery-oriented mental health system of care. Theresa manages MHA's programs including MHA Screening, The State of Mental Health in America, and Workplace Wellness. She oversees MHA's research which explores the integration of peers into research, the use of technology to support people in the earliest stages of recovery, and how large-scale data provides insight into gaps in supports systems across the country. As a Licensed Clinical Social Worker, she has over 15 years of experience in mental health as a clinician, educator, and advocate. Her clinical experience focused on working with children and adults with serious mental illness, homelessness, dual diagnosis treatment, and early intervention of psychosis. As an advocate she worked to build a consumer based mental health workforce, to improve access to treatment through community based and recovery oriented mental health programs, and to address needs of underserved communities. She is an adjunct professor in California and has taught courses covering Mental Health Recovery, Psychosocial Rehabilitation and Social Welfare Policy. Prior to joining MHA National, Theresa worked at both MHALA (Los Angeles, CA) and MHAOC (Orange County, CA).

John A. Naslund is Instructor of Global Health and Social Medicine at Harvard Medical School. He holds expertise in psychiatric epidemiology, implementation science, social disparities research and digital mental health. His scholarship seeks to advance efforts aimed at improving the lives of individuals facing the challenges of mental illness worldwide. Specifically, his work explores the intersection between emerging digital technologies, such as smartphones, mobile applications, social media, AI, and web-platforms, and implementation science, towards improving the availability, reach, quality and effectiveness of mental health services. Dr. Naslund has a longstanding track record advocating for the rights, dignity, and quality of healthcare for those living with mental illness. 